\title{
Fractional Integrals Involving Generalized Polynomials And Multivariable Function
}

\author{
Dr Neelam Pandey and Reshma Khan \\ Department of Mathematics APS university Rewa- 486001 Madhya Pradesh,India
}

Abstract:Our aim of this paper is to find a Eulerian Integral and a main theorem based on the fractional operator associated with generalized polynomial and a multivariable I-function having general arguments. The theorem provides extension of various results. Some special cases are also given.

Keywords-Fractionalintegral,Eulerian integral,multivariable I-function,Riemann-Liouville operator,Lauricella function.

\section{Introduction}

The Riemann-Liouville operator of fractional integration $\mathrm{R}^{\mathrm{m}} \mathrm{f}$ of order $\mathrm{m}$ is defined by $x D_{y}^{-m}[f(y)]=\frac{1}{\Gamma(\mathrm{m})} \int_{x}^{y}(y-t)^{m-1} \cdot f(t) \cdot d t$

An equivalent form of Beta function is

$\int_{m}^{n}(t-m)^{a-1} \cdot(n-t)^{b-1} \cdot d t=(n-m)^{a+b-1} B(a, b)$

Where $m, n \in R(x<y), \operatorname{Re}(a)>0, \operatorname{Re}(b)>0$

To prove the Eulerian integrals, we use the following formula.

$\int_{x}^{y}(t-x)^{a-1} \cdot(y-t)^{b-1} \cdot\left(p_{1} t+q_{1}\right)^{\rho_{1}} \ldots \ldots\left(p_{h} t+q_{h}\right)^{\rho_{h}} d t=(y-x)^{a+b-1} \cdot B(a, b) \cdot\left(p_{1} x+q_{1}\right)^{e_{1}} \ldots \ldots\left(p_{h} x+\right.$ qh)eh

$\times F_{D}^{(h)}\left[a,-\rho_{1} \ldots .-\rho_{h} ; a+b, \frac{-(y-x) \rho_{1}}{p_{1} x+q_{1}} \ldots \ldots \frac{-(y-x) \rho_{h}}{p_{h} x+q_{h}}\right]$

Where $F_{D}^{(h)}$ is Lauricella function

Where $\mathrm{x}, \mathrm{y} \in \mathrm{R}(\mathrm{x}<\mathrm{y}) ; p_{j}, q_{j} \rho_{j} \in C(j=1 \ldots \ldots h)$

$\min \left[R_{e}(a), R_{e}(b)\right]>0$ and $\left.\max \left|\frac{(y-x) \rho_{1}}{P_{1} x+q_{1}}\right|, \ldots \ldots\left|\frac{(y-x) \rho_{h}}{P_{h} x+q_{1 h}}\right|\right]<1$

The integral representation of $F_{D}^{(h)}$ is defined as

$$
\begin{array}{r}
\frac{\Gamma(a) \cdot \Gamma\left(b_{1}\right) \ldots \ldots \ldots \ldots\left(b_{h}\right)}{\Gamma(\mathrm{c})} \cdot F_{D}^{(h)}\left[\mathrm{a}, \mathrm{b}_{1} \ldots \ldots \ldots \mathrm{b}_{\mathrm{h}}, \mathrm{c}, \mathrm{x}_{1} \ldots \ldots \mathrm{x}_{\mathrm{h}}\right]= \\
\frac{1}{(2 \pi \mathrm{i})^{\mathrm{h}}} \int_{-\mathrm{i} \infty}^{\mathrm{i} \infty} \ldots h \ldots \int_{-\mathrm{i} \infty}^{\mathrm{i} \infty} \frac{\Gamma\left(a+\xi_{1}+\ldots \xi_{h}\right) \cdot \Gamma\left(b+\xi_{1}\right) \ldots \ldots \ldots \Gamma\left(b+\xi_{h}\right)}{\Gamma\left(\mathrm{c}+\xi_{1}+\ldots \xi_{h}\right)}, \Gamma\left(-\xi_{1}\right) \ldots . \Gamma\left(-\xi_{\mathrm{h}}\right) \times \\
\left(-x_{1}\right)^{\xi_{1}} \ldots \ldots\left(-x_{h}\right)^{\xi_{h}} d \xi_{1} \ldots . . d \xi_{h}
\end{array}
$$

Now $\left(p_{1} t+q\right)^{\alpha}=(x p+q)^{\alpha} \cdot\left[1+\frac{p(t-x)}{x p+q}\right]^{\alpha}=(x p+q)^{\alpha} \cdot 2 F_{1}\left[-\alpha, 1,1, \frac{-p(t-x)}{x p+q}\right]$

Since $\frac{\Gamma(\mathrm{A}) \cdot \Gamma(\mathrm{B})}{\Gamma(c)} \cdot 2 F_{1}[A, B, C ; Z]=\frac{1}{2 \pi \mathrm{i}} \int_{-i \infty}^{i \infty} \frac{\Gamma(\mathrm{A}+\zeta) \cdot \Gamma(\mathrm{B}+\zeta)}{\Gamma(c+\zeta)} \Gamma(-\zeta) \cdot(-Z) \zeta d \zeta$

$\therefore,(p t+q)^{\alpha}=$

$\frac{(x p+q)^{\alpha}}{\Gamma(-\alpha)} \frac{1}{2 \pi \mathrm{i}} \int_{-i \infty}^{i \infty} \Gamma(-\zeta) \cdot \Gamma(\zeta-\alpha) \cdot\left\{\frac{p(t-x)}{x p+q}\right\} \stackrel{\zeta}{\zeta} d \zeta$

$\mathrm{p}, \mathrm{q}, \alpha \in C, x, t \in R$ and $\left|\arg \left(\frac{p}{x p+q}\right)\right|<\pi$ and path of integration is necessary in such a manner so as to separate the poles of $\Gamma(-\varsigma)$ from those if $\Gamma(\varsigma-\alpha)$.

Formula (3), can be provided with the help of (2), (4) and (5)

The generalized polynomial defined by Shrivastava[8] is as follows 


$$
\begin{aligned}
& S_{N_{1} \ldots \ldots . . N_{k}}^{M_{1}}\left[x_{1} \ldots \ldots \ldots . x_{k}\right] \\
& =\sum_{\alpha_{1}=0}^{\left[N_{1} / M_{1}\right]} \ldots \ldots \ldots \sum_{\alpha_{k}=0}^{\left[N_{k} / M_{k}\right]} \frac{\left(-N_{1}\right) M_{1} \alpha_{1}}{\left\llcorner\alpha_{1}\right.} \ldots \frac{\left(-N_{k}\right) M_{k} \alpha_{k}}{\left\llcorner\alpha_{k}\right.} B\left[N_{1}, \alpha_{1}, \ldots \ldots, N_{k}, \alpha_{k}\right] x_{1}{ }^{\alpha_{1}} \ldots \ldots x_{k}{ }^{\alpha_{k}}
\end{aligned}
$$

Where, $N_{i}=0,1,2, \ldots \ldots \forall i=(1 \ldots \ldots \ldots k), M_{1} \ldots \ldots M_{k}$ are arbitrary positive integers and the coefficients $B\left[N_{1}, \alpha_{1}, \ldots \ldots, N_{k}, \alpha_{k}\right]$ are arbitrary constants.

Here $\mathrm{h}$ is a positive integer and $0 \ldots \ldots \ldots . .0$ would mean $\mathrm{h}$ zeros.

\section{MULTI-VARIABLE I-FUNCTION}

It is defined and represented in the following manner:-

$$
\begin{aligned}
& I\left[z_{1} \ldots \ldots z_{1}\right]=I_{p_{2}, q_{2}: p_{3}, q_{3}, \ldots . ., p_{r}, q_{r}, p^{1}, q^{1} \ldots \ldots . . .}^{0, n_{2}: p^{(r)}, q^{(r)}}
\end{aligned}
$$

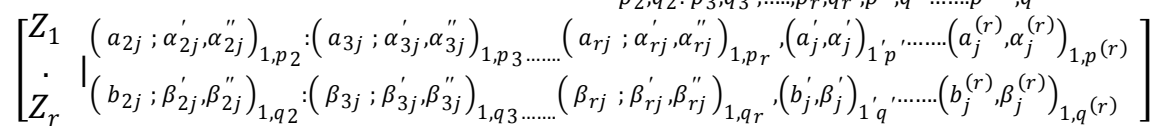

$$
\begin{aligned}
& =\left(\frac{1}{2 \pi \omega}\right)^{r} \cdot \int_{L_{1}} \ldots . \int_{L_{1}} \phi\left(\xi_{1}\right) \ldots \ldots \varnothing\left(\xi_{r}\right) . \Psi\left(\xi_{1} \ldots \ldots \xi_{r}\right) \cdot z_{1}^{\xi_{1}} \ldots \ldots \mathrm{z}_{\mathrm{r}}^{\xi_{\mathrm{r}}} \mathrm{d} \xi_{1} \ldots \ldots \mathrm{d} \xi_{\mathrm{r}}
\end{aligned}
$$

$$
\begin{gathered}
\text { Where, } \phi_{\mathrm{i}}\left(\xi_{\mathrm{i}}\right)=\frac{\prod_{j=1}^{m_{i}} \Gamma\left(b_{j}^{(i)}-\beta_{j}^{(i)} \xi_{i}\right) \prod_{j=1}^{n_{i}} \Gamma\left(1-a_{j}^{(i)}+\alpha_{j}^{(i)} \xi_{i}\right)}{\prod_{j=m^{(i)}+1}^{q_{i}} \Gamma\left(1-b_{j}^{(i)}+\beta_{j}^{(i)} \xi_{i}\right) \prod_{j=n^{(i)}+1}^{p^{(i)}} \Gamma\left(a_{j}^{(i)}-\alpha_{j}^{(i)} \xi_{i}\right)} \text { and } \\
\psi\left(\xi_{1} \ldots \xi_{r}\right)=\frac{\prod_{j=1}^{n_{2}} \Gamma\left(1-a_{2 j}+\sum_{i=1}^{2} \alpha_{2 j}^{(i)} \xi_{i}\right) \prod_{j=1}^{n_{3}} \Gamma\left(1-a_{3 j}+\sum_{i=1}^{3} \alpha_{3 j}^{(i)} \xi_{i}\right) \ldots \prod_{j=1}^{n_{r}} \Gamma\left(1-a_{r j}+\sum_{i=1}^{r} \alpha_{r j}^{(i)} \xi_{i}\right)}{\prod_{j=n_{2}+1}^{p_{2}} \Gamma\left(a_{2 j}-\sum_{i=1}^{2} \alpha_{2 j}^{(i)} \xi_{i}\right) \prod_{j=n_{3}+1}^{p_{3}} \Gamma\left(a_{3 j}-\sum_{i=1}^{3} \alpha_{3 j}^{(i)} \xi_{i}\right) \ldots \prod_{j=n_{r}+1}^{p_{r}} \Gamma\left(a_{r j}-\sum_{i=1}^{r} \alpha_{r j}^{(i)} \xi_{i}\right)}
\end{gathered}
$$$$
\times \frac{1}{\prod_{j=1}^{q_{2}} \Gamma\left(1-b_{2 j}+\sum_{i=1}^{2} \beta_{2 j}^{(i)} \xi_{i}\right) \ldots \prod_{j=1}^{q_{r}} \Gamma\left(1-b_{r j}+\sum_{i=1}^{r} \beta_{r j}^{(i)} \xi_{i}\right)}
$$

The convergence and other details of multivariable I-function, see Prasad[4].

\section{Main Integral}

The main Integral to be established here is

$$
\begin{gathered}
\int_{m}^{n}(t-m)^{a-1}(n-t)^{b-1} \cdot\left\{\prod_{j=1}^{h}\left(p_{j}+q_{j}\right)^{\rho_{j}}\right\} \\
S_{N_{1} \ldots . . . N_{k}}^{M_{1} M_{k}}\left[\begin{array}{c}
x_{1}(t-m)^{\lambda_{1}}(n-t)^{\mu_{1}} \cdot \prod_{j=1}^{h}\left(p_{j} t+q_{j}\right)^{\sigma_{j}^{1}} \\
\cdot \\
\cdot \\
x_{k}(t-m)^{\lambda_{k}}(n-t)^{\mu_{k}} \cdot \prod_{j=1}^{h}\left(p_{j} t+q_{j}\right)^{\sigma_{j}^{k}}
\end{array}\right] \cdot I\left[\begin{array}{c}
z_{1}(t-m)^{\gamma_{1}}(n-t)^{\tau}{ }_{1 \cdot \prod_{j=1}^{h}\left(p_{j} t+q_{j}\right)^{-c_{j}^{1}}} \\
\cdot \\
\cdot \\
z_{r}(t-m)^{\gamma_{r}} \\
\gamma_{(n-t)} \tau_{r \cdot \prod_{j=1}^{h}\left(p_{j} t+q_{j}\right)^{-c_{j}^{r}}}
\end{array}\right] d t=
\end{gathered}
$$

$W_{1} \sum_{\alpha_{1}=0}^{\left[\frac{N_{1}}{M_{1}}\right]} \ldots \ldots \sum_{\alpha_{k}=0}^{\left[\frac{N_{k}}{M_{k}}\right]} \frac{\left(-N_{1}\right)_{M_{1} \alpha_{1}}}{! \alpha_{1}} \ldots \ldots \frac{\left(-N_{k}\right)_{M_{k} \alpha_{k}}}{! \alpha_{k}}$. 


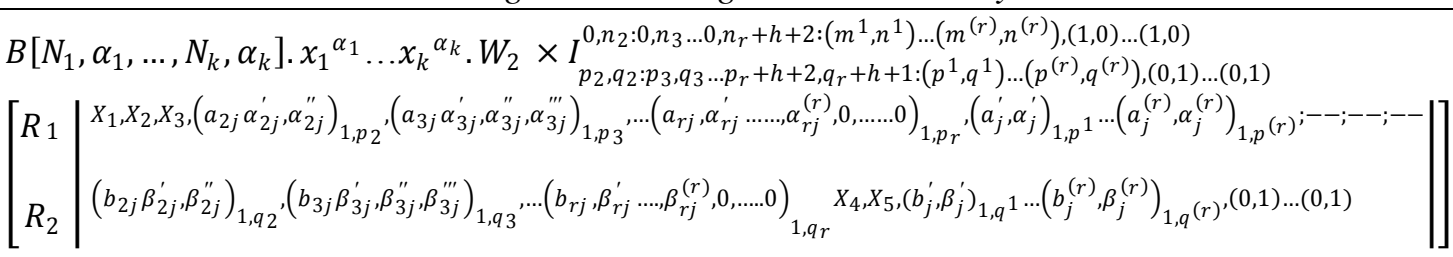

Where $W_{1}=(n-m)^{a+b-1}\left\{\prod_{j=1}^{h}\left(p_{j} m+q_{j}\right)^{p_{j}}\right\}$

$W_{2}=(n-m)^{\sum_{i=1}^{k}\left(\lambda_{i}+\mu_{i}\right) \alpha_{i}} \cdot\left\{\prod_{j=1}^{h}\left(p_{j} m+q_{j}\right)^{\sum_{i=1}^{k} \sigma_{j}^{(i)} \cdot \alpha_{i}}\right\}$

$X_{1}=\left[1-a-\sum_{i=1}^{k} \lambda_{i} . \alpha_{i} ; \gamma_{i} \ldots \ldots \gamma_{r, 1 \ldots \ldots .1}\right]$

$X_{2}=\left[1-b-\sum_{i=1}^{k} \mu_{i} \cdot \alpha_{i} ; \tau_{1} \ldots \ldots \tau_{r, 0 \ldots \ldots .}\right]$

$X_{3}=\left[1+\rho_{j}+\sum_{i=1}^{k} \sigma_{j}^{(i)} \cdot \alpha_{i} ; C_{j}^{1} \ldots \ldots C_{j}^{(r)}, 0 \ldots \ldots .1 \ldots \ldots .0\right]_{1, h}$

$X_{4}=\left[1+\rho_{j}+\sum_{i=1}^{k} \sigma_{j}^{(i)} \cdot \alpha_{i} ; C_{j}^{1} \ldots \ldots C_{j}^{(r)}, 0 \ldots \ldots \ldots \ldots .0\right]_{1, h}$

$X_{5}=\left[1-a-b-\sum_{i=1}^{k}\left(\lambda_{i}+\mu_{i}\right) . \alpha_{i} ; \gamma_{1}+\tau_{1} \ldots \ldots, 1 \ldots \ldots \gamma_{r}+\tau_{r}, 1 \ldots 1\right]$

$R_{1}=\left\{\begin{array}{c}Z_{1}(n-m)^{\gamma_{1}+\tau_{1}} / \prod_{j=1}^{h}\left(p_{j} m+q_{j}\right)^{c_{j}^{1}} \\ \vdots \\ Z_{r}(n-m)^{\gamma_{r}+\tau_{r}} /_{\prod_{j=1}^{h}\left(p_{j} m+q_{j}\right)^{c_{j}^{(r)}}}\end{array}\right.$

$R_{2}=\left\{\begin{array}{l}\frac{(n-m) p_{1}}{p_{1} m+q_{1}} \\ \cdot \cdot \\ \frac{(n-m) p_{h}}{p_{h} m+q_{h}}\end{array}\right.$

The following are the conditions of validity of integral (8)

1) $\quad m, n \in R(m<n), \gamma_{i}, \tau_{i} ; c_{j}^{(i)}, \lambda_{i}, \mu_{i}, \sigma_{j}^{(i)} \in R^{+}, \rho_{j} \in R p_{j}, q_{j} \in C, z_{i} \in C(i=1 \ldots \ldots ., j=1 \ldots . . h)$

2) $\max _{1 \leq j \leq h}\left[\left|\frac{(\mathrm{n}-\mathrm{m}) \mathrm{p}_{\mathrm{j}}}{\mathrm{p}_{\mathrm{j}} m+q_{j}}\right| \mid<1\right.$

3) $\quad R_{e}\left[a+\sum_{i=1}^{r} \gamma_{i} \frac{b_{j}^{(i)}}{B_{j}^{(i)}}\right]>0, j=1 \ldots m^{(i)}, R_{e}\left[b+\sum_{i=1}^{r} \tau_{i} \frac{b_{j}^{(i)}}{B_{j}^{(i)}}\right]>0, j=1 \ldots . m^{(i)}$ 
4) $\quad\left|\arg \left(z_{i}\right) \prod_{j=1}^{h}\left(p_{j}+q_{j}\right)^{-c_{j}^{i}}\right|<\frac{T_{i} \pi}{2}(m \leq t \leq n, i=1 \ldots \ldots r)$

Where

$$
\begin{aligned}
T_{i}=\sum_{j=1}^{n^{(i)}} \alpha_{j}^{(i)}- & \sum_{j=n^{(i)}+1}^{p^{(i)}} \alpha_{j}^{(i)}+\sum_{j=1}^{m^{(i)}} \beta_{j}^{(i)}-\sum_{j=m^{(i)}+1}^{q^{(i)}} \beta_{j}^{(i)}+\left(\sum_{j=1}^{n_{2}} \alpha_{2_{j}}{ }^{(i)}-\sum_{j=n_{2}+1}^{p_{2}} \alpha_{2_{j}}{ }^{(i)}\right) \\
& +\left(\sum_{j=1}^{n_{3}} \alpha_{3_{j}}{ }^{(i)}-\sum_{j=n_{3}+1}^{p_{3}} \alpha_{3_{j}}{ }^{(i)}\right)+\cdots+\left(\sum_{j=1}^{n_{r}} \alpha_{r_{j}}{ }^{(i)}-\sum_{j=n_{r}+1}^{p_{r}} \alpha_{r_{j}}{ }^{(i)}\right) \\
& +\left(\sum_{j=1}^{q_{2}} \beta_{2_{j}}{ }^{(i)}+\sum_{j=1}^{q_{3}} \beta_{3_{j}}{ }^{(i)}+\cdots+\sum_{j=1}^{q_{r}} \beta_{r_{j}}{ }^{(i)}\right)
\end{aligned}
$$

\section{PROOF}

In order to prove integral (8),expand multivariable I-function in terms of mellin-Barnes type of contour integral by (7), generalized polynomial by(6).Now interchanging the order of summation and integration (which is permissible under the conditions of validity stated above), We get the following form:-

$$
\begin{aligned}
& \sum_{\alpha_{1}=0}^{\left[{ }^{N_{1}} / M_{1}\right]} \ldots \ldots . \sum_{\alpha_{k}=0}^{\left[{ }^{N_{k}} / M_{k}\right]} \frac{\left(-N_{1}\right)_{M_{1} \alpha_{1}}}{! \alpha_{1}} \ldots \ldots \frac{\left(-N_{k}\right)_{M_{k} \alpha_{k}}}{! \alpha_{k}} B\left[N_{1} \alpha_{1}, \ldots \ldots, N_{k} \alpha_{k}\right] . x_{1}{ }^{\alpha_{1}} \ldots \ldots x_{k}{ }^{\alpha_{k}} \\
& X\left(\frac{1}{2 \pi \mathrm{w}}\right)^{\mathrm{r}} \int_{\mathrm{L}_{1}} \ldots \ldots \int_{\mathrm{L}_{\mathrm{r}}} \emptyset_{1}\left(\xi_{1}\right) \ldots \ldots \emptyset_{\mathrm{r}}\left(\xi_{\mathrm{r}}\right) \cdot \mathrm{Z}_{1}^{\xi_{1}} \ldots \ldots \mathrm{Z}_{\mathrm{r}} \xi_{\mathrm{r}}\left\{\int_{\mathrm{m}}^{\mathrm{n}}(\mathrm{t}-\mathrm{m})^{\mathrm{a}+\sum_{\mathrm{i}=1}^{\mathrm{k}} \lambda_{\mathrm{i}} \mathrm{d}_{\mathrm{i}}+\sum_{\mathrm{s}}^{\mathrm{r}}=1 \gamma_{\mathrm{s}} \xi_{\mathrm{s}}-1} \cdot(\mathrm{n}\right.
\end{aligned}
$$

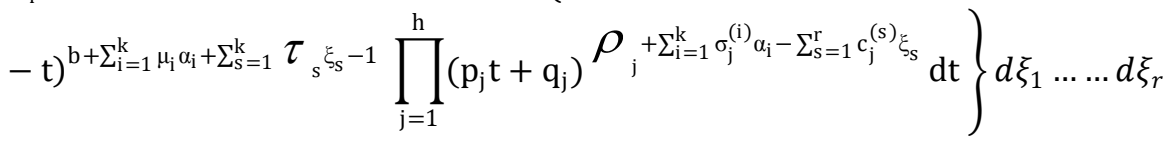

Now using the formula (3) for inner integral i.e,

$\int_{x}^{y}(t-x)^{a-1} \cdot(y-t)^{b-1} \cdot\left(p_{1} t+q_{1}\right)^{\rho_{1}} \ldots . .\left(p_{h} t+q_{h}\right)^{\rho_{k}} d t=$ $y-x a+b-1 \cdot B a, b \cdot(p 1 x+q 1) e 1 \ldots \ldots(p h x+q h) e n \quad \times F D(h)$

$a,-\rho 1 \ldots-\rho h$

$; a+b$

$-y-x p 1 p 1 x+q 1 \ldots \ldots-y-x p h p h x+q h$

And converting the Lauricella function $F_{D}^{(h)}$ in integral form from equation (4) and after simplification, we get the required result:

IV. SPACIAL CASES:-

1.If we set $\gamma_{1}=0=\cdots=\gamma_{r}$ and $\lambda_{1}=0=\cdots=\lambda_{k}$, the integral (8) reduces to

$$
\left.\begin{array}{c}
\int_{m}^{n}(t-m)^{a-1}(n-t)^{b-1} \cdot\left\{\prod_{j=1}^{h}\left(p_{j} t+q_{j}\right)^{\rho_{j}}\right\} \\
S_{N_{1} \ldots N_{k}}^{M_{1} \ldots M_{k}} \\
x_{1}(n-t)^{\mu_{1}} \cdot \prod_{j=1}^{h}\left(p_{j} t+q_{j}\right)^{\sigma_{j}^{1}} \\
\cdot \\
x_{k}(n-t)^{\mu_{k}} \cdot \prod_{j=1}^{h}\left(p_{j} t+q_{j}\right)^{\sigma_{j}^{k}}
\end{array}\right] \cdot I\left[\begin{array}{c}
z_{1}(n-t)^{\tau_{1}} \cdot \prod_{j=1}^{h}\left(p_{j} t+q_{j}\right)^{-c_{j}^{1}} \\
\cdot \\
z_{r}(n-t)^{2} \tau_{r} \cdot \prod_{j=1}^{h}\left(p_{j} t+q_{j}\right)^{-c_{j}^{r}}
\end{array}\right]
$$

$=E_{1} \sum_{\alpha_{1}=0}^{\left[\frac{N_{1}}{M_{1}}\right]} \ldots$

$\sum_{\alpha_{k}=0}^{\left[\frac{N_{k}}{M_{k}}\right]} \frac{\left(-N_{1}\right)_{M_{1} \alpha_{1}}}{! \alpha_{1}} \ldots \ldots \frac{\left(-N_{k}\right)_{M_{k} \alpha_{k}}}{! \alpha_{k}} . B\left[N_{1}, \alpha_{1}, \ldots, N_{k}, \alpha_{k}\right] \cdot x_{1}^{\alpha_{1}} \ldots x_{k}{ }^{\alpha_{k}} \cdot E_{2} \times$

$I^{0, n_{2}: 0, n_{3} \ldots 0, n_{r}+h+2:\left(m^{1}, n^{1}\right) \ldots\left(m^{(r)}, n^{(r)}\right),(1,0) \ldots(1,0)}$

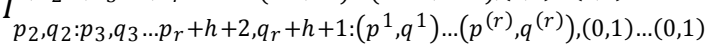




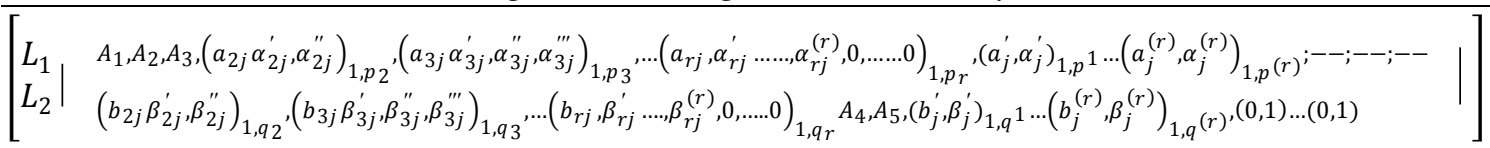

Where, $W_{1}=(n-m)^{a+b-1}\left\{\prod_{j=1}^{h}\left(p_{j} m+q_{j}\right)^{\rho_{j}}\right\}$

$W_{2}=(n-m)^{\sum_{i=1}^{k} \mu_{i} \cdot \alpha_{i}} \cdot\left\{\prod_{j=1}^{h}\left(p_{j} m+q_{j}\right)^{\sum_{i=1}^{k} \sigma_{j}^{(i)}} \cdot \alpha_{i}\right\}$

$A_{1}=[1-a ; \overbrace{0 \ldots \ldots 0}^{r}, 1 \ldots \ldots 1]$

$A_{2}=\left[1-b-\sum_{i=1}^{k} \mu_{i} . \alpha_{i} ; \zeta_{i} \ldots \ldots \zeta_{r, 0 \ldots \ldots 0}\right]$

$A_{3}=\left[1+\rho_{j}+\sum_{i=1}^{k} \sigma_{j}^{(i)} \cdot \alpha_{i} ; C_{j}^{1} \ldots \ldots C_{j}^{(r)}, 0 \ldots \ldots 1^{j} \ldots \ldots .0\right]_{1, h}$

$A_{4}=\left[1+\rho_{j}+\sum_{i=1}^{k} \sigma_{j}^{(i)} \cdot \alpha_{i} ; C_{j}^{1} \ldots \ldots C_{j}^{(r)}, 0 \ldots \ldots 1^{j} \ldots \ldots .0\right]_{1, h}$

$A_{5}=\left[1-a-b-\sum_{i=1}^{k} \mu_{i} . \alpha_{i} ; \zeta_{i} \ldots \ldots \zeta_{r}, 1 \ldots \ldots .1\right.$

$L_{1}=\left\{\begin{array}{c}Z_{1}(n-m)^{\zeta_{1}} / \prod_{j=1}^{h}\left(p_{j} m+q_{j}\right)^{c_{j}^{1}} \\ Z_{r}(n-m)^{\zeta_{r}} / \\ \prod_{j=1}^{h}\left(p_{j} m+q_{j}\right)^{c_{j}^{(r)}}\end{array}\right.$

$L_{2}=\left\{\begin{array}{l}\frac{(n-m) p_{1}}{p_{1} m+q_{1}} \\ \cdot \cdot \\ \frac{(n-m) p_{h}}{p_{h} m+q_{h}}\end{array}\right.$

2. For $\zeta_{1}=0=\cdots=\zeta_{r}$ and $\mu_{1}=0=\cdots \mu_{k}$, the integral (8) reduces to

$$
\int_{m}^{n}(t-m)^{a-1}(n-t)^{b-1} \cdot\left\{\prod_{j=1}^{h}\left(p_{j}+q_{j}\right)^{\rho_{j}}\right\}
$$

$S_{N_{1} N_{k}}^{M_{1} M_{k}}\left[\begin{array}{cc}x_{1}(t-m)^{\lambda_{1}} & \prod_{j=1}^{h}\left(p_{j} t+q_{j}\right)^{\sigma_{j}^{1}} \\ \cdot & \cdot \\ x_{k}(t-m)^{\lambda_{k}} & \prod_{j=1}^{h}\left(p_{j} t+q_{j}\right)^{\sigma_{j}^{k}}\end{array}\right] . I\left[\begin{array}{cc}z_{1}(t-m)^{\gamma_{1}} & \prod_{j=1}^{h}\left(p_{j} t+q_{j}\right)^{-c_{j}^{1}} \\ \cdot \\ \cdot \\ z_{r}(t-m)^{\gamma_{k}} & \prod_{j=1}^{h}\left(p_{j} t+q_{j}\right)^{-c_{j}^{r}}\end{array}\right]=\Gamma(b) F_{1} \sum_{\alpha_{1}=0}^{\left[\frac{N_{1}}{M_{1}}\right]} \ldots \ldots$

$\sum_{\alpha_{k}=0}^{\left[\frac{N_{k}}{M_{k}}\right]} \frac{\left(-N_{1}\right)_{M_{1} \alpha_{1}}}{! \alpha_{1}} \ldots \ldots \frac{\left(-N_{k}\right)_{M_{k} \alpha_{k}}}{! \alpha_{k}} \cdot B\left[N_{1}, \alpha_{1}, \ldots, N_{k}, \alpha_{k}\right] \cdot x_{1}{ }^{\alpha_{1}} \ldots x_{k}{ }^{\alpha_{k}} \cdot F_{2} \times$

$I^{0, n_{2}: 0, n_{3} \ldots 0, n_{r}+h+1:\left(m^{1}, n^{1}\right) \ldots\left(m^{(r)}, n^{(r)}\right),(1,0) \ldots(1,0)}$

$I_{p_{2}, q_{2}: p_{3}, q_{3} \ldots p_{r}+h+1, q_{r}+h+1:\left(p^{1}, q^{1}\right) \ldots\left(p^{(r)}, q^{(r)}\right),(0,1) \ldots(0,1)}$ 


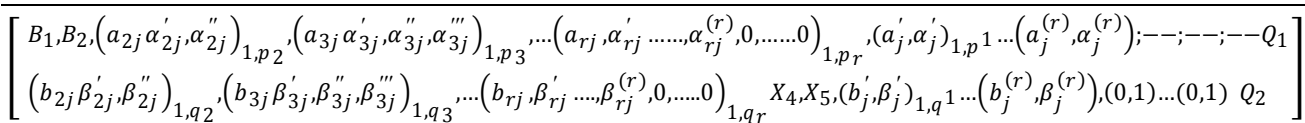

Where, $F_{1}=(n-m)^{a+b-1}\left\{\prod_{j=1}^{h}\left(p_{j} m+q_{j}\right)^{\rho_{j}}\right\}$

$F_{2}=(n-m)^{\Sigma_{i=1}^{k} \lambda_{i} \alpha_{i}} \cdot\left\{\prod_{j=1}^{h}\left(p_{j} m+q_{j}\right)^{\sum_{i=1}^{k} \sigma_{j}^{(i)} \cdot \alpha_{i}}\right\}$

$B_{1}=\left[1-a-\sum_{i=1}^{k} \lambda_{i} \cdot \alpha_{i} ; \gamma_{i} \ldots \ldots \gamma_{r, 1 \ldots \ldots .1}\right]$

$B_{2}=\left[1+\rho_{j}+\sum_{i=1}^{k} \sigma_{j}^{(i)} \cdot \alpha_{i} ; C_{j}^{1} \ldots \ldots C_{j}^{(r)}, 0 \ldots \ldots 1 \ldots \ldots .0\right]_{1, h}$

$B_{3}=\left[1+\rho_{j}+\sum_{i=1}^{k} \sigma_{j}^{(i)} \cdot \alpha_{i} ; C_{j}^{1} \ldots \ldots C_{j}^{(r)}, 0 \ldots \ldots \ldots \ldots .0\right]_{1, h}$

$B_{4}=\left[1-a-b-\sum_{i=1}^{k} \lambda_{i} \alpha_{i} ; \gamma_{i} \ldots \ldots \gamma_{r}, 1 \ldots \ldots .1\right.$

$Q_{1}=\left\{\begin{array}{c}Z_{1}(n-m)^{\gamma_{1}} / \prod_{j=1}^{h}\left(p_{j} m+q_{j}\right)^{c_{j}^{1}} \\ \vdots \\ Z_{r}(n-m)^{\gamma_{r}} / \prod_{j=1}^{h}\left(p_{j} m+q_{j}\right)^{c_{j}^{(r)}}\end{array}\right.$

$Q_{2}=\left\{\begin{array}{l}\frac{(n-m) p_{1}}{p_{1} m+q_{1}} \\ \cdot \\ \frac{(n-m) p_{h}}{p_{h} m+q_{h}}\end{array}\right.$

3. When $\zeta_{1}=0=\cdots=\zeta_{r}=0=\gamma_{1}=\cdots \gamma_{r}$ and $\lambda_{i}=0=\mu_{i},(i=1 \ldots \ldots k)$, then integral (8) reduces to

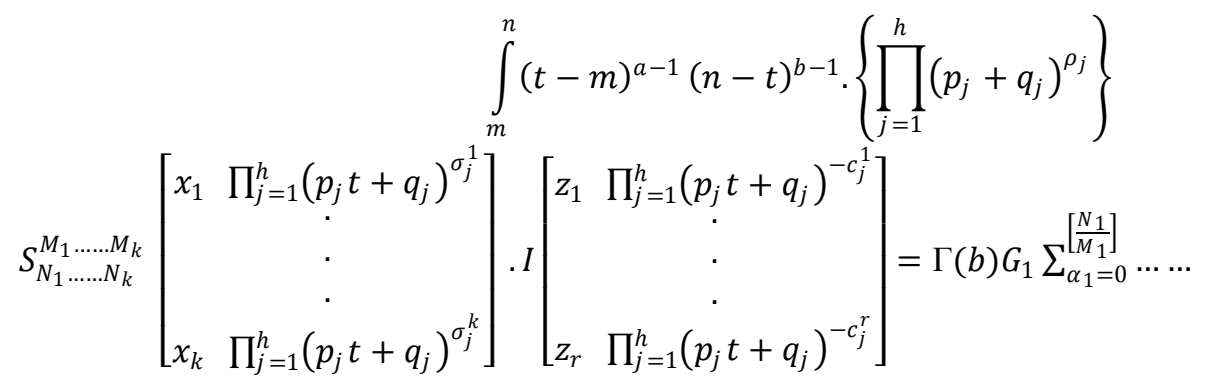

$\sum_{\alpha_{k}=0}^{\left[\frac{N_{k}}{M_{k}}\right]} \frac{\left(-N_{1}\right)_{M_{1} \alpha_{1}}}{! \alpha_{1}} \ldots \ldots \frac{\left(-N_{k}\right)_{M_{k} \alpha_{k}}}{! \alpha_{k}} . B\left[N_{1}, \alpha_{1}, \ldots, N_{k}, \alpha_{k}\right] \cdot x_{1}{ }^{\alpha_{1}} \ldots x_{k}{ }^{\alpha_{k}} \cdot G_{2} \times$

$I^{0, n_{2}: 0, n_{3} \ldots 0, n_{r}+h+1:\left(m^{1}, n^{1}\right) \ldots\left(m^{(r)}, n^{(r)}\right),(1,0) \ldots(1,0)}$

$p_{2}, q_{2}: p_{3}, q_{3} \ldots p_{r}+h+1, q_{r}+h+1:\left(p^{1}, q^{1}\right) \ldots\left(p^{(r)}, q^{(r)}\right),(0,1) \ldots(0,1)$

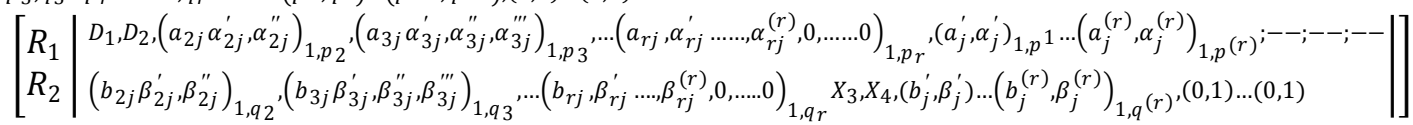


Where, $G_{1}=(n-m)^{a+b-1}\left\{\prod_{j=1}^{h}\left(p_{j} m+q_{j}\right)^{\rho_{j}}\right\}$

$$
\begin{aligned}
& G_{2}=\left\{\prod_{j=1}^{h}\left(p_{j} m+q_{j}\right)^{\sum_{i=1}^{k} \sigma_{j}^{(i)} \cdot \alpha_{i}}\right\} \\
& D_{1}=[1-a ; \overbrace{0 \ldots \ldots .}^{r}, 1 \ldots \ldots .1] \\
& D_{2}=\left[1+\rho_{j}+\sum_{i=1}^{k} \sigma_{j}^{(i)} \cdot \alpha_{i} ; C_{j}^{1} \ldots \ldots C_{j}^{(r)}, 0 \ldots \ldots 1 \ldots \ldots .0\right] \\
& D_{3}=\left[1+\rho_{j}+\sum_{i=1}^{k} \sigma_{j}^{(i)} \cdot \alpha_{i} ; C_{j}^{1} \ldots \ldots C_{j}^{(r)}, 0 \ldots \ldots \ldots \ldots .0\right] \\
& D_{4}=[1-a-b ; \overbrace{0 \ldots \ldots}^{r}, 1 \ldots \ldots .1 \\
& R_{1}=\left\{\begin{array}{l}
Z_{1} \cdot \prod_{j=1}^{h}\left(p_{j} m+q_{j}\right)^{c_{j}^{1}} \\
Z_{r} \cdot \prod_{j=1}^{h}\left(p_{j} m+q_{j}\right)^{c_{j}^{(r)}}
\end{array}\right. \\
& R_{2}=\left\{\begin{array}{l}
\frac{(n-m) p_{1}}{p_{1} m+q_{1}} \\
\frac{(n-m) p_{h}}{p_{h} m+q_{h}}
\end{array}\right.
\end{aligned}
$$

\section{MAIN THEOREM}

Let $f(t)=(t-m)^{a-1}\left\{\prod_{j=1}^{h}\left(p_{j} t+q_{j}\right)^{\rho_{j}}\right\}$.

Then, ${ }_{m} D_{y}^{-b}\{f(y)\}=\frac{1}{\Gamma(\mathrm{b})} \cdot \int_{m}^{y}(y-t)^{b-1} \cdot f(t) d t$

$$
S_{N_{1} N_{k} M_{k}}^{M_{1}}\left[\begin{array}{cc}
x_{1}(t-m)^{\lambda_{1}} & \prod_{j=1}^{h}\left(p_{j} t+q_{j}\right)^{\sigma_{j}^{1}} \\
\cdot & \cdot \\
x_{k}(t-m)^{\lambda_{k}} & \prod_{j=1}^{h}\left(p_{j} t+q_{j}\right)^{\sigma_{j}^{k}}
\end{array}\right] \cdot I\left[\begin{array}{c}
z_{1}(t-m)^{\gamma_{1}} \prod_{j=1}^{h}\left(p_{j} t+q_{j}\right)^{-c_{j}^{1}} \\
\cdot \\
z_{r}(t-m)^{\gamma_{k}} \prod_{j=1}^{h}\left(p_{j} t+q_{j}\right)^{-c_{j}^{r}}
\end{array}\right]
$$

$$
\begin{aligned}
& { }_{m} D_{y}^{-b}[f(y)] \cdot \frac{1}{\Gamma(\mathrm{b})} \cdot \int_{m}^{y}(t-m)^{a-1}(y-t)^{b-1} \cdot\left\{\prod_{j=1}^{h}\left(p_{j} t+q_{j}\right)^{\rho_{j}}\right\} \\
& S_{N_{1} \ldots N_{k}}^{M_{1} \ldots M_{k}}\left[\begin{array}{cc}
x_{1}(t-m)^{\lambda_{1}} & \prod_{j=1}^{h}\left(p_{j} t+q_{j}\right)^{\sigma_{j}^{1}} \\
\cdot & \cdot \\
x_{k}(t-m)^{\lambda_{k}} & \prod_{j=1}^{h}\left(p_{j} t+q_{j}\right)^{\sigma_{j}^{k}}
\end{array}\right] . I\left[\begin{array}{c}
z_{1}(t-m)^{\gamma_{1}} \prod_{j=1}^{h}\left(p_{j} t+q_{j}\right)^{-c_{j}^{1}} \\
\cdot \\
\cdot \\
z_{r}(t-m)^{\gamma_{k}} \prod_{j=1}^{h}\left(p_{j} t+q_{j}\right)^{-c_{j}^{r}}
\end{array}\right]
\end{aligned}
$$




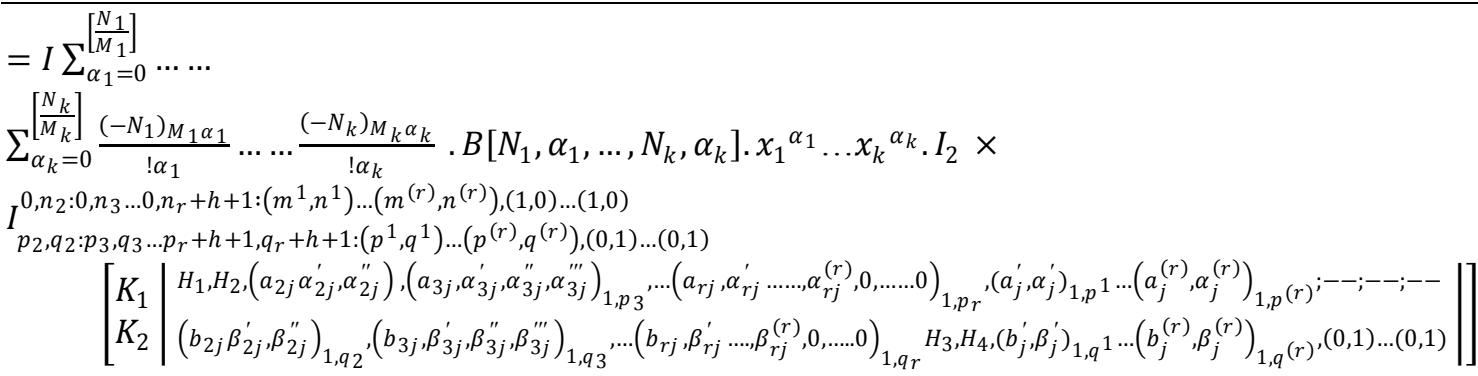

Where, $G_{1}=(y-m)^{a+b-1}\left\{\prod_{j=1}^{h}\left(p_{j} m+q_{j}\right)^{\rho_{j}}\right\}$

$G_{2}=(y-m)^{\sum_{i=1}^{k} \lambda_{i} \alpha_{i}}\left\{\prod_{j=1}^{h}\left(p_{j} m+q_{j}\right)^{\sum_{i=1}^{k} \sigma_{j}^{(i)} \cdot \alpha_{i}}\right\}$

$H_{1}=\left[1-a-\sum_{i=1}^{k} \lambda_{i} \cdot \alpha_{i} ; \gamma_{i} \ldots \ldots \gamma_{r, 1 \ldots \ldots 1}\right]$

$H_{2}=\left[1+\rho_{j}+\sum_{i=1}^{k} \sigma_{j}^{(i)} \cdot \alpha_{i} ; C_{j}^{1} \ldots \ldots C_{j}^{(r)}, 0 \ldots \ldots 1 \ldots \ldots .0\right]_{1, h}$

$H_{3}=\left[1+\rho_{j}+\sum_{i=1}^{k} \sigma_{j}^{(i)} \cdot \alpha_{i} ; C_{j}^{1} \ldots \ldots C_{j}^{(r)}, 0 \ldots \ldots \ldots \ldots\right]_{1, h}$

$H_{4}=\left[1-a-b-\sum_{i=1}^{k} \lambda_{i} \alpha_{i} ; \gamma_{1} \ldots \ldots \gamma_{r}, 1 \ldots \ldots .1\right.$

$K_{1}=\left\{\begin{array}{c}Z_{1}(y-m)^{\gamma_{1}} / \prod_{j=1}^{h}\left(p_{j} m+q_{j}\right)^{c_{j}^{1}} \\ Z_{r}(y-m)^{\gamma_{r}} / \\ \prod_{j=1}^{h}\left(p_{j} m+q_{j}\right)^{c_{j}^{(r)}}\end{array}\right.$

$K_{2}=\left\{\begin{array}{l}\frac{(y-m) p_{1}}{p_{1} m+q_{1}} \\ \cdot \cdot \\ \frac{(y-m) p_{h}}{p_{h} m+q_{h}}\end{array}\right.$

Conditions of validity of the theorem are the same as stated in Eulerian Integral.

Special case

By specializing the various parameters, we get some known and unknown results.

\section{Refrences}

[1]. Y.N. Prasad, Multivariable I-Function, Vijnana Parishad Anusandhan Patrika 29(1986) 231 - 235

[2]. H. M. Shrivastava and M A Hussain, Fractional integration of the H-Function of several variable, compute, Math, April 30(1995),73-85

[3]. V.B.L Chaurasia and V.K Singhal,Fractional integration of certain special functions,Tamkang J.Math.35(2004),13-22.

[4]. A.P.Prudnikov,Yu.A. Brychkov and O.I.Marichev,Integrals and series,Vol.I,Elementary Functions, Gordon and Breach,NewyorkLondon-Paris-Montreux-Tokyo, 1986.

[5]. M. Saigo and R.K.Saxena ,Unified fractional integral formula for the multivariable H-function,J.Fract.Calc.15 (19999),91-107.

[6]. R.K. Saxena and K.Nishimoto,Fractional integral formula for the H-function,J.Fract.Calc. 13 (1994),65-74.

[7]. R.K. Saxena and M. Saigo, Fractional integral formula for the H-function II,J.Fract.Calc. 6 (1994),37-41.

[8]. H.M.Srivastava and M.C.Daoust,Certain generalized Neumann expansions associated with the Kampe de Feriet function,Nederl.Acad.We-tench.Indag.Math. 31 (1969),449-457.

[9]. H.M. Srivastava , K.C. Gupta and S.P. Goyal,The H-functions of One and Two Variables with Applications,South Asian Publishers,New Delhi-Madras,1982. 